\title{
Primary small-cell neuroendocrine carcinoma of the bladder: Case report and literature review
}

\author{
Valerio Olivieri ${ }^{1}$, Valentina Fortunati ${ }^{2}$, Luca Bellei $^{1}$, Massimo Massarelli ${ }^{1}$, Gabriele Ruggiero ${ }^{3}$, \\ Danilo Abate $^{3}$, Nicoletta Serra ${ }^{3}$, Daniele Griffa ${ }^{1,3}$, Flavio Forte ${ }^{4}$, Emanuele Corongiu ${ }^{4}$ \\ ${ }^{1}$ Division of Urology, Ivrea Hospital (ASL TO4), Ivrea (Turin), Italy; \\ ${ }^{2}$ Division of Pathology, Hospital Policlinico of Tor Vergata, Rome, Italy; \\ ${ }^{3}$ Division of Urology, Ciriè Hospital (ASLTO4), Ciriè (Turin), Italy; \\ ${ }^{4}$ Division of Urology, Hospital "Madre Giuseppina Vannini", Rome, Italy.
}

\begin{abstract}
Summary Background: Neuroendocrine tumours (NET) are extremely rare and aggressive. Although they commonly affect intestine, many organs may be involved such as pancreas, lung or urinary tract. Bladder is rarely involved. Actually, two main forms of bladder NET have been described: small-cell and large-cell. The first one is considered highly agressive since it shows poor oncologic outcomes being mainly diagnosed at advanced stage: the second one is extremely rare and equally aggressive.

Case report: A 78-years-old Caucasian male presented to our facility for lower urinary tract symptoms and gross hematuria recently occurred. He was a strong smoker since many years. No familiarity for urothelial cancer was referred nor previous episodes of hematuria until that time. Citology was negative; outpatient ultrasound of the bladder revealed a $3 \mathrm{~cm}$ bladder thickening highly suspicious for bladder cancer; patient underwent TC scan that confirmed the bladder lesion.

A transurethral resection of the bladder (TURB) was performed. After 3 months total body TC showed multiple visceral metastases also involving brain and lymph nodes. Best supportive care was offered but the patient died 6 months later. Results: Pathology revealed a mixed bladder tumor: $30 \%$ of the specimen resulted as an high-grade urothelial cancer (G3) and $70 \%$ as small-cell neuroendocrine variant.Microscopic muscle involvement was excluded.

Conclusions: Neuroendocrine tumors are uncommon entities which origin from cells of neuro-endocrine system and may potentially involve all human tissues. Neuroendocrine smallcell carcinoma of the bladder is a non-urothelial histotype: it is highly aggressive and diagnosed mainly at advanced stages. Whenever considering the high risk of metastatic spread and the poor prognosis, a multimodal approach is highly suggested. TURB alone is uneffective in disease control due to its aggressive nature. Unless metastatic, radical cystectomy and adjuvant chemotherapy represent the gold standard.
\end{abstract}

KEY WORDS: Neuroendocrine; Bladder cancer; Uro-oncology; Rare tumors; Oncology.

Submitted 2 March 2020; Accepted 15 March 2020

\section{INTRODUCTION}

Neuroendocrine tumours (NET) are extremely rare (1) and aggressive. It has been valued the annual incidence is approximately 2.5-5.0 per 100.000.

They take origin from cells of nervous and endocrine systems differently expressed in human tissues. Although they commonly affect intestine, many organs may be involved such as pancreas, lung or urinary tract.

Bladder is rarely involved (2). To date two main forms of bladder NET have been described: small-cell and largecell. The first one is considered highly aggressive since it shows poor oncologic outcomes being mainly diagnosed at advanced stage: the second one is extremely rare and equally aggressive. Data in literature are strongly limited. Here we report the case of a small-cell neuroendocrine carcinoma of urinary bladder. While opportunely describing this case, we also reviewed the literature on PubMed about this pathological condition.

\section{Case report}

A 78-years-old Caucasian male presented to our facility for lower urinary tract symptoms and gross hematuria recently occurred. He was a strong smoker since many years. No familiarity for urothelial cancer was referred nor previous episodes of haematuria until that time.

On past anamnesis he suffered from myocardial infarction and chronic fibrillation being actually on medication with acetilsalicilate plus edoxaban. Multiple coexisting systemic illness were reported such as hypertension, dyslipidemia and mild chronic renal failure.

Furthermore, he was also on medication with adrenergic al-antagonist for a benign prostatic hyperplasia (BPH) condition: anyway, his last PSA was normal and digito-rectal exploration confirmed an enlarged soft gland. General and systemic examination was unremarkable: no asthenia, weight loss, abdominal palpable masses or acute urinary retention were present. Blood examination revealed a mild anaemia while renal function and white blood count were unremarkable. Urinalysis confirmed haematuria but cytology was negative. Ambulatory ultrasound revealed a $3 \mathrm{~cm}$ bladder wall thickening highly suspicious for bladder cancer; the patient subsequently underwent to contrastenhanced CT. The examination confirmed the bladder lesion (Figure 1) totally excluding bilateral hydronephrosis, visceral metastases or lymph nodes involvement.

A trans urethral resection (TURB) of the mass was offered. Pathology report revealed a mixed bladder tumour: 30\% of specimen resulted as an high-grade urothelial cancer (G3) while $70 \%$ as small-cell neuroendocrine variant (Figure 2). Microscopic muscle involvement was excluded. In order to better characterize the neuroendocrine differentiation, further evaluation using immunohistochemistry was mandatory: cytokeratin, synaptophysin and 


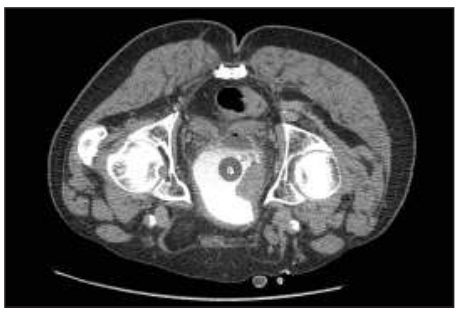

Figure 1.

Abdominal contrast enhanced CT showing a right wall bladder lesion.

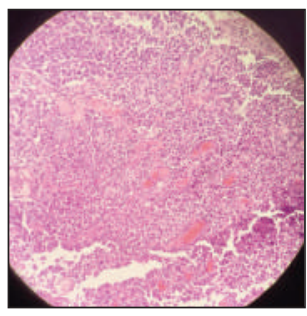

Figure 2.

Microscopic finding showing small cell neuroendocrine bladder cancer reacting to synaptophysin (original magnification x 400).

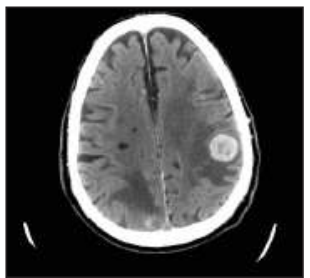

Figure 3-4.

Whole body contrast enhanced CT showing brain and liver multiple metastases in small cell neuroendocrine carcinoma of the bladder (SCCB).
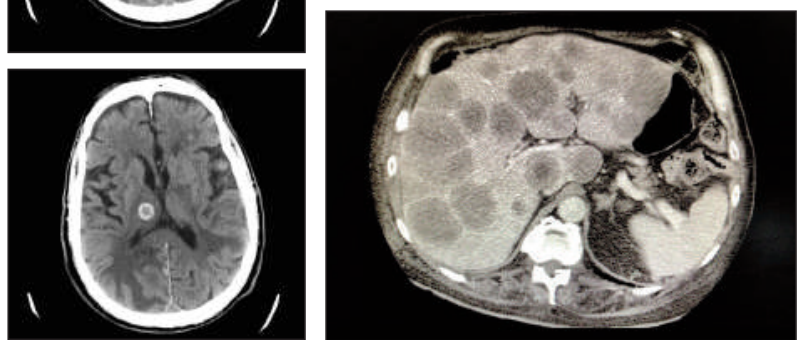

Ki-67 were used as molecular markers. Urothelial histotype resulted positive for cytokeratine but negative for synaptophysin while the neuroendocrine variant was positive for both of them. Ki-67, a molecular marker as expression of proliferation rate, was $90 \%$ totally suggesting an aggressive bladder cancer, in fact NET are histologically graded according to mitotic count and markers of cellular proliferation (Ki-67 index) rather than cellular polymorphism: a mitotic count $>20$ per $10 \mathrm{HPF}$ and a Ki-67 index $>20 \%$ defines the high-grade. Due to its non-urothelial origins, it was also classified as a highest risk tumour. Treatments, in these cases, depends on many factors; several issues such as anatomic location, tumour differentiation, staging, metastases, lymph node involvement and symptoms may help to define the best therapeutic approach. The high-grade forms should be managed by active treatment which may be multimodal. Unless metastatic, radical cystectomy and chemotherapy (either neoadjuvant or adjuvant) must be offered (3) since TURB alone seems to be an inadequate method of disease control. Recently, some authors noted molecular abnormalities in small cell neuroendocrine bladder cancer also raising the question of using target therapies: targeting angiogenesis would be the most promising. Anyway, to date immunotherapy still remains under debate. In our case, we evaluated the patient in a multidisciplinary tumour board in order to offer the best disease management. According to Karnofsky performance status, comorbidities and iatrogenic bleeding risk, adjuvant chemotherapy and radiation were not suggested: radical cystectomy was also offered but patient refused that option. He simply underwent follow up. After 3 months total body TC showed multiple visceral metastases (Figures 3-4) also involving brain and lymph nodes: best supportive care was offered but the patient died 6 months later.

\section{Conclusions}

Neuroendocrine tumours are uncommon entities l which origin from cells of neuro-endocrine system and may potentially involve all human tissues.

Gastrointestinal localization is quite common while genitourinary is rare. Neuroendocrine small-cell carcinoma of the bladder is a non-urothelial histotype: it is highly aggressive and diagnosed mainly at advanced stages. It's considered as an aggressive tumor being characterized by poor prognosis and limited life expectancy. Small-cell carcinoma is a type of bladder NET along with large-cell variant. To date no treatment has shown established efficacy. Whenever considering the high risk of metastatic spread and the poor prognosis, a multimodal approach is highly suggested. TURB alone is uneffective in disease control due to its aggressive nature.

Unless metastatic, radical cystectomy and adjuvant chemotherapy represent the gold standard. Immunotherapy has been proposed but still under debate. Given its rarity and the absence of randomized trials, the therapeutic management of this tumor remains difficult and finding useful resources for physicians is troublesome yet.

\section{REFERENCES}

1. Al-Ahmadie H, Iyer G. Updates on the genetics and molecular subtypes of urothelial carcinoma and select variants. Surg Pathol Clin. 2018; 11:713-723

2. Cramer SF, Aikawa M, Cebelin M. Neurosecretory granules in small cell invasive carcinoma of the urinary bladder. Cancer. 1981; 47:724-30

3. Siefker-Radtke AO, Dinney CP, Abrahams NA, et al. Evidence supporting preoperative chemotherapy for small cell carcinoma of the bladder: a retrospective review of the M.D. Anderson cancer experience. J Urol. 2004; 172:481-484.

\author{
Correspondence \\ Valerio Olivieri, MD \\ valerio.olivieri@uniromal.it \\ Luca Bellei, MD \\ Massimo Massarelli, MD \\ Gabriele Ruggiero, MD \\ Division of Urology, Ivrea Civil Hospital (ASL TO4), Ivrea (Turin) (Italy) \\ Daniele Griffa, MD \\ Danilo Abate, MD \\ Nicoletta Serra, MD \\ Division of Urology, Ciriè Hospital (ASLTO4), Ciriè (Turin) (Italy) \\ Valentina Fortunati, $M D$ \\ valerio.olivieri@uniromal.it \\ Division of Pathology, Hospital "Policlinico of Tor Vergata", Rome (Italy) \\ Emanuele Corongiu, MD (Corresponding Author) \\ emanuele.corongiu@libero.it \\ Flavio Forte, MD \\ flavioforte@hotmail.com \\ Division of Urology, Hospital "Madre Giuseppina Vannini", Rome (Italy)
}

